\title{
A WIDE FIELD $\lambda$ AOCM IMAGE AROUND THE GALACTIC CENTRE - EVIDENCE FOR A POLOIDAL MAGNETIC FIELD
}

\author{
K.R. Anantharamaiah, Raman Research Institate, Bangalore, India \\ A. Pedlar, Nuffield Radio Astronomy Laboratories, Jodrell Bank, U.K.
}

\section{Introduction}

A number of unique non-thermal filamentary structures, which are all roughly perpendicular to the galactic plane have been discovered in the vicinity of the Galactic centre (see Yusef-Zadeh 1989 and references there in). On the basis of their uniformity, polarization angles, rigidity, and pressure balance with the surrounding medium, it has been hypothesized that a strong polodial field of milligauss strength pervades the inner $50 \mathrm{pc}$ of the Galaxy (Morris and Yusef-Zadeh 1989 and Morris in this volume). We present here a single wide-field image, at $\lambda 90 \mathrm{~cm}$, which shows all the filamentary structures in this region and show that these observations are consistent with the above hypothesis.

\section{Observations}

The observations were obtained in the B, C, and D configurations of the Very Large Arrayt (VLA) at a frequency of $333.1 \mathrm{MHz}$ and a bandwidth of $\sim 1.25 \mathrm{MHz}$. The combined, self-calibrated and cleaned image, which has a resolution of $\mathbf{3 3}^{\prime \prime} \times 17^{\prime \prime}$ is shown in Figure 1. In addition to the SgrA complex at the centre, this image clearly shows the Arc, "threads", and other filamentary structures, which have been discovered at higher frequencies (cf Yusef-Zadeh 1989).

\section{The poloidal magnetic field}

Whereas the mechanism for production of the unique filamentary structures seen in Figure 1 is not understood, there is broad agreement that such structures must be a consequence of a substantial poloidal magnetic filed (Morris and Yusef-Zadeh 1989 and references there in) in the central region of the Galaxy. The present observations reinforce this hypothesis, and the suggestion of a poloidal field is particularly compelling in Figure 1, where the filamentary structures could be envisaged to be part of a dipole field. Although the minimum energy approximation (Miley 1980) is almost certainly invalid in the Arc and the threads, it can be used to set conservative lower limits to the magnetic field. Assuming that the depth of the filaments are equal to their mean widths, and that the energies in relativistic protons and electrons are equal, we obtain lower limits in the range $4.2-9.5 \times 10^{-6} \mathrm{G}$ for the isolated filaments seen in Figure 1. Yusef-Zadeh and Morris (1987) have argued that the field strength must exceed $10^{-3} \mathrm{G}$ in the Arc.

† The VLA is part of the National Radio Astronomy Observatory, which is operated by the Associated Universities Inc., under the cooperative agreement with the NSF 


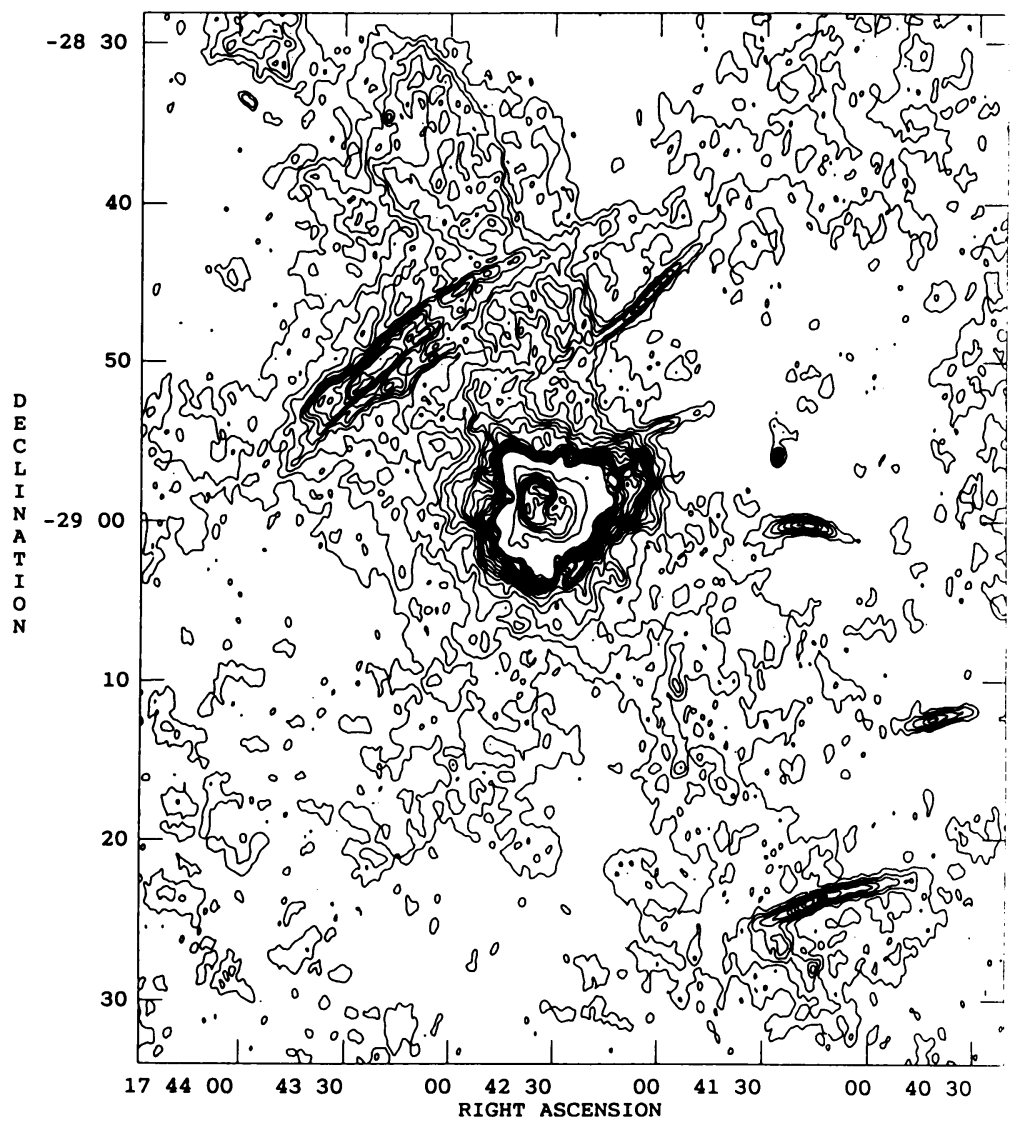

Figure 1. A $\lambda 90 \mathrm{~cm}$ image around the Galactic centre. The angular resolution is $33^{\prime \prime} \times 17^{\prime \prime}$. The contour levels are $-25,25$ to 500 in steps of 25, 1000 to 5000 in steps of $500 \mathrm{mJy} /$ beam.

The origin of this field is unclear, although it could imply the existence of a Galactic dynamo. It is possible that a mechanism similar to the one proposed by Lesch et al (1989) for M82, albeit on a smaller scale, could be operating in our Galactic centre. As yet the evidence for such a dynamo is largely circumstantial.

\section{References}

Lesch, H., et al, 1989, this volume

Miley, G., 1980, Ann. Rev. Astr. Ap., 18, 165

Morris, M., and Yusef-Zadeh, F., 1989, Ap. J., 343, 703

Yusef-Zadeh, F., and Morris, M., 1987, A.J., 94, 1178

Yusef-zadeh, F., 1989, in The Center of the Galaxy, ed. M. Morris

Kluwer Academic Publishers, Dordrecht 